\title{
Hábitos alimenticios y dieta invernal de Charadrius melodus (Charadriiformes: Charadriidae) en Boca Ciega, Tamaulipas, México
}

Iris Banda-Villanueva ${ }^{1}$, Jorge Contreras-Lozano ${ }^{2}$, Juan García-Salas ${ }^{1} \&$ Hugo González-Páez ${ }^{1}$

1. Laboratorio de Ornitología, Facultad de Ciencias Biológicas, Universidad Autónoma de Nuevo León. Apartado Postal 425 San Nicolás de los Garza, Nuevo León, CP 66450, México; i_a_b_v@hotmail.com,juan_0305@hotmail.com, halcon14hg@hotmail.com

2. Laboratorio de Herpetología Facultad de Ciencias Biológicas, Universidad Autónoma de Nuevo León. Apartado Postal 425 San Nicolás de los Garza, Nuevo León, CP 66450, México; jorgeacl.84@gmail.com

Recibido 13-II-2012. Corregido 20-VIII-2012. Aceptado 24-IX-2012.

\begin{abstract}
Food habits and winter diet of Charadrius melodus (Charadriiformes: Charadriidae) in Boca Ciega, Tamaulipas, Mexico. The Piping Plover (Charadrius melodus) is a migratory endangered species that arrives, along with a great number of other winter migratory birds, to Boca Ciega every year. In spite of the importance of this ecosystem, these species, are threatened by the current habitat change caused by the dredging activities in the area. With the aim to generate new information about the importance of this area during winter, we studied C. melodus activities during the winter season in Laguna Madre, from December 2009 to March 2010. Our objectives were: 1) determine the importance of the area during winter, 2) describe C. melodus ethology, feeding substrate preferences and food items, 3 ) to analyze and describe the sympatric diversity associated with C. melodus. A total of ninety nine individuals were observed during the monitoring. The Cochran and Kendall test showed a high significance of the species with the substrate and signs tests using a binomial distribution that indicated a high preference for algal type of substrate. The highest activity recorded for this species during this winter season was feeding. The principal food items found in sediments were larvae of Diptera: Chironomidae and Ephydridae. The sympatric species of $C$. melodus were two families of Charadriiforms: Scolopacidae (nine species) and Charadriidae (two species). We concluded that this is an important area for feeding, protection and rest sites for this species, and its protection and management is recommended. Rev. Biol. Trop. 61 (2): 841-848. Epub 2013 June 01.
\end{abstract}

Key words: Piping plover, Boca Ciega, Charadriidae, Tamaulipas, Laguna Madre, Mexico.

Uno de los ecosistemas más importantes de la Provincia Biótica Tamaulipeca es la Laguna Madre; que se extiende desde el estado de Louisiana en los Estados Unidos de Norteamérica hasta el Río Soto la Marina, Tamaulipas, México (Labougle et al. 2002). Ocupa una extensión aproximada de $9055.09 \mathrm{~km}^{2}$ y es considerado el cuerpo de agua continental más grande de México. La Laguna Madre tiene gran importancia ecológica y económica, ya que es un área de refugio, crianza, reproducción y alimentación de numerosas especies entre las que destacan las aves acuáticas, además de mantener la mayor pesquería comercial y recreativa del Norte de México (DUMAC 1993, Farmer \& Carrera 1993, Labougle et al. 2002).

Invernan el $15 \%$ del total de las aves migratorias que arriban a México provenientes de Canadá y Estados Unidos de Norteamérica, al encontrar sitios de protección, alimentación y anidación. Entre las especies que sobresalen, está el pato cabeza roja (Aythya americana), la única colonia reproductora de pelícano blanco (Pelecanus erythrorhynchos) en ambientes costeros de México, sin mencionar una numerosa cantidad de patos, gansos y aves playeras. Entre los sitios más importantes del ecosistema para las aves acuáticas están Higuerillas, 
Laguna Jasso, así como los islotes de Boca Ciega y las islas del sur de la Laguna Madre (DUMAC 1993).

C. melodus se distribuye en Estados Unidos y Canadá, y pasa el invierno en Carolina del Norte y Carolina del Sur (EUA), Cuba, las Bahamas y las Islas Turcas y Caicos, con algunos registros en Jamaica, Barbados, Haití, República Dominicana, Puerto Rico, Islas Vírgenes y San Cristóbal y Nieves (BirdLife International 2008). Para México, se ha observado en Veracruz, noreste de Yucatán, noreste de Quintana Roo, Puerto Peñasco, Sonora, San Blas y Nayarit (Haig \& Elliott-Smith 2004).

Algunos de estos estudios se han enfocado en conocer la distribución y el uso del hábitat (Ridway 1919, Cairns \& McLaren 1980, Adams 1984, Haig \& Oring 1985, Nicholls \& Baldassarre 1990a, Boyd 1991, Zonick 2000, Drake et al. 2001), factores demográficos (Haig \& Oring 1987, Haig \& Oring 1988a, 1988b, Prindiville-Gaines \& Ryan 1988, Schwalbach 1988, MacIvor 1990, Patterson et al. 1991, Root et al. 1991, Powell \& Cuthbert 1992), caracterización de hábitats (Brown 1987, Johnson \& Baldassarre 1988, PrindivilleGaines \& Ryan 1988, Nicholls \& Baldassarre 1990b, Nordstrom 1990, Melvin et al. 1991, Sidle et al. 1992, Ziewitz et al. 1992), determinación de los efectos de los disturbios humanos (MacIvor 1990, Strauss 1990, Flemming et al. 1988), y la dieta. Bent (1929) encontró gusanos marinos, insectos, crustáceos, moluscos y otros animales marinos. Nicholls (1989) en heces fecales moluscos, anélidos, artrópodos, crustáceos y nematodos. Shaffer \& Laporte (1994) a partir de observaciones en playas y bahías, encontraron que se alimentaban de poliquetos. Zonick (2000) en sustrato con trampas de pegamento observó anfípodos. Nordstrom \& Ryan (1996) citan que la dieta está compuesta principalmente por dípteros de las familias Chironomidae y Ephydridae.

El estatus de la especie en México se encuentra en peligro de extinción de acuerdo con la NOM-059-SEMARTNAT-2010 (SEMARNAT 2010). La Unión Internacional para la Conservación la incluye en la lista roja desde 1988, primero como amenazada y posteriormente como vulnerable en 1994, 2000, 2004, y casi amenazada 2001, 2005, 2006 hasta la actualidad (BirdLife International 2008).

Las poblaciones de $C$. melodus se encuentran amenazadas por la destrucción o degradación de sitios de anidación, la depredación de nidos, y el disturbio humano (Flemming et al. 1988, Haig \& Oring 1988a, PrindivilleGaines \& Ryan 1988). En 1950 se detectó el descenso de las poblaciones encontrándose bajo la categoría de peligro de extinción para la NOM-059-SEMARNAT-2010 (SEMARNAT 2010), condición que ha incrementado un mayor interés de investigadores y organizaciones, para estudiar y entender las razones de dicho problema. Por lo anterior, es importante incrementar el conocimiento sobre esta especie durante el invierno, ya que puede pasar de siete a ocho meses lejos de las áreas de reproducción (Haig \& Oring 1985). El presente trabajo tiene como objetivo conocer la distribución, el comportamiento y dieta de la especie, en este sitio de Tamaulipas, en su periodo invernal del 2009-2010, con el fin de generar las estrategias adecuadas para la conservación y manejo adecuado del área para la especie.

\section{MATERIALES Y MÉTODOS}

El área de estudio se encuentra en una porción de la Laguna Madre comprendida en el Municipio de San Fernando, Tamaulipas, México (Martínez 1978, SPP 1983). La Laguna Madre es hipersalina ( $\mathrm{S}=42 \mathrm{o} / \mathrm{oo}$ promedio anual), a excepción de su extremo sur donde se presentan características estuarinas por la influencia del Río Soto la Marina (Zabalegui [XE “Zabalegui, M.L.M.”] \& Contreras 1990). El área de estudio se compone de dos zonas: norte y sur, las cuales están separadas por un canal (iniciado en el 2008 por el proyecto construcción de escolleras y dragado) para el paso de lanchas al mar abierto. La diferencia principal entre la zona norte y sur es una barrera de arena (producto del dragado) que limita la introducción de agua a la zona sur. Las coordenadas geográficas del polígono que se trabajó 
son: zona norte $25^{\circ} 02^{\prime} 0.65^{\prime \prime} \mathrm{N}-97^{\circ} 32^{\prime} 49.92^{\prime \prime}$ $\mathrm{W}$ and $25^{\circ} 00^{\prime} 26.58^{\prime \prime} \mathrm{N}-97^{\circ} 31^{\prime} 53.91^{\prime \prime} \mathrm{W}$ y zona sur $25^{\circ} 01^{\prime} 1.09^{\prime \prime} \mathrm{N}-97^{\circ} 33^{\prime} 17.21^{\prime \prime} \mathrm{W}$ and $25^{\circ} 00^{\prime} 7.68^{\prime \prime} \mathrm{N}-97^{\circ} 32^{\prime} 5.17^{\prime}$ 'W. El área estudiada tiene una extensión territorial de $8.8 \mathrm{~km}^{2}$ aproximadamente. El sitio es una planicie, delimitada por dunas de arena que se encuentran en la parte norte y sur.

Presenta un clima semiseco muy cálido, con una temperatura promedio anual de $22.4^{\circ} \mathrm{C}$. La precipitación promedio anual es $38 \mathrm{~mm}$ con ciertas variaciones (INEGI 2000). Los frentes fríos ejercen una gran influencia en los meses de octubre-marzo con los vientos llamados "nortes" con velocidades entre $25-85 \mathrm{~km} / \mathrm{h}$ y su frecuencia de ocurrencia es de 25-40 frentes fríos por año (INEGI 2000). La fase de marea varía muy lentamente en el Golfo de México por lo que la hora de la bajamar y la pleamar se presenta casi simultáneamente en toda la zona (Lanza-Espino 1991).

El muestreo fue mensual durante la temporada migratoria de invierno de C. melodus se realizó entre diciembre 2009 y marzo 2010. Cada salida tuvo una duración de tres a cuatro días. Los conteos se realizaron entre las 800-1 $300 \mathrm{~h}$ y $1500-1800 \mathrm{~h}$, invirtiendo ocho horas de esfuerzo de trabajo. La información obtenida durante cada salida fue in situ, datando condiciones ambientales como nubosidad, temperatura, humedad y velocidad promedio del viento mediante un anemómetro LT-ABH4224. Para las especies simpátricas se registró la fecha, hora, nombre, número de individuos, área de observación y actividad. Para los individuos de $C$. melodus se tomaron datos conductuales como: alimentación (A), vuelo (V) y descanso (D) y tipo de sustrato donde se encontraba. El sustrato se clasificó en algal: cuando los individuos eran observados en superficies húmedas y firmes, de apariencia verdosa, seguidas de una capa delgada de lodo; mud: cuando estaban en superficies húmedas pardas y muy suaves seguidas de una capa de aproximadamente $4 \mathrm{~cm}$ de lodo; y sand, cuando las superficies húmedas eran amarillas.

La observación de las aves se realizó en tierra con binoculares Bushnell (10X50) y Pentax (10X40), un monocular VORTEX Skyline 20-60x80 Angled Spotting Scope SKL- $80^{\text {a }}$ y otro monocular Bushnell spacemaster 20-60X60mm. El arreglo sistemático de las especies se realizó de acuerdo al criterio de la American Ornithologist Union (1998). Cada observación fue georefrenciada con la ayuda de un GPS GARMIN Etrex Legend $\mathrm{H}$ y se descargaron sobre una imagen satelital de Boca Ciega, Tamaulipas, México.

Para confirmar la preferencia de Charadrius melodus por un tipo de sustrato, se realizó la prueba de Cochran-Q y de Kendall-W. Además, se utilizaron pruebas de signos para determinar la hipótesis de que la preferencia del C. melodus es la misma para los tres sustratos, mediante el programa SPSS ver. 15 (IBM Corporation Somers, New York, USA).

Se empleó una modificación de la técnica de Zonick (2000) para conocer la dieta, se tomó un total de 15 muestras del sedimento donde se observó la especie forrajeando en suelo, utilizando una pala de metal. Por cada individuo de C. melodus, se tomó cinco muestras aleatorias de sedimento dentro de un radio de $3 \mathrm{~m}$ del punto de donde fue observado el individuo. $\mathrm{Si}$ se observaba más de un individuo se obtenían 15 muestras de sedimento. Las medidas de cada muestra fueron de $5 \mathrm{~cm}$ de diámetro por $5 \mathrm{~cm}$ de profundidad. Se eligió esta profundidad debido a que la longitud del pico es corta, aproximadamente de 11.0-13.5mm de largo. Una vez tomadas las muestras de sedimento, se introdujeron en bolsas de plástico y se vertió formol al $10 \%$ hasta cubrir el sedimento para fijar el material. Después de dos días, se reemplazó el formol por alcohol etílico al 70\% para conservar las muestras; posteriormente, las mismas se lavaron usando un tamiz de $425 \mu$ (micras), y luego, proceder a determinar los organismos hasta familia siguiendo el criterio de Merritt \& Cummins (1996) mediante un estereoscopio Leica ZOOM2000 Model No.Z30V 120Vac.

\section{RESULTADOS}

Se registraron un total de 99 individuos de $C$. melodus durante los meses de diciembre 
2009-marzo 2010, distribuidos de la siguiente manera: $21.21 \%$ corresponden en la primera salida, $19.19 \%$ en la segunda, $47.47 \%$ en la tercera, y el $12.12 \%$ en la cuarta, respectivamente (Cuadro 1).

La mayor actividad de $C$. melodus se presentó entre las 10:00-15:00h, donde fueron observados 58 de los 99 individuos. En general, el tiempo fue utilizado siempre para alimentarse; y no se observó actividad reproductiva durante el muestreo. La técnica de forrajeo empleada por esta especie fue la visual, aunque en ocasiones se observó usando sus patas para la búsqueda del alimento.

La mayoría de los individuos (80 individuos) se observaron en el sustrato tipo algal, seguido del mud (17 individuos) y en menor proporción en el sand ( 2 individuos). Las pruebas de Cochran-Q y Kendall-W resultaron altamente significativas hacia un tipo de sustrato $(\mathrm{Q}=103.818, \mathrm{p}=0.000)$; mientras que el coeficiente de concordancia $(\mathrm{W}=0.514, \mathrm{p}=0.000$, donde $\mathrm{p}>0.00$ ) indicó que la especie tiende a elegir más un tipo de sustrato que otro. La prueba de signos utilizando una distribución binomial indicó que las preferencias de la especie hacia los tres tipos de sustrato no es la misma, donde los valores de las comparaciones entre los tres sustratos resultó: Algal-Sand $=0.001$, Mud-Sand $=0.000$, Mud-Algal $=0.000$.

Por su parte, se pudo revisar 15 muestras de sustrato para el estudio de la dieta, que correspondieron a individuos alimentándose, además se tomaron dos muestras tomadas en

\section{CUADRO 1}

Datos de población, actividad, sustrato y parámetros ambientales obtenidos durante los muestreos de Charadrius melodus en diciembre 2009-marzo 2010 en el área de Boca Ciega, Tamaulipas, México. Abreviaturas: Ind.- número de individuos, A- alimentándose, V- vuelvo, D- descanso, Temp.- temperatura, HR- humedad relativa, (-) no determinado

TABLE 1

Data of population, activity, substrate and environmental parameters, obtained during the sampling of Charadrius melodus on December 2009-March 2010 in Boca Ciega, Tamaulipas, Mexico. Abbreviations: Ind.- specimens, A- feeding, V- flying, D- quiet, Temp.- temperature, HR- relative humidity, (-) not determined

\begin{tabular}{|c|c|c|c|c|c|c|c|}
\hline Salida & Área & Ind. & Actividad & Temp ${ }^{\circ} \mathrm{C}$ & Substrato & HR (\%) & Viento $(\mathrm{km} / \mathrm{h})$ \\
\hline Diciembre & Norte & 1 & A V & 23 & Mud & 67 & - \\
\hline Diciembre & Norte & 1 & A V & 22.3 & Mud & 64 & - \\
\hline Diciembre & Norte & 2 & A & 23 & Algal & 55 & - \\
\hline Diciembre & Sur & 1 & A & 12.5 & Algal & 71 & - \\
\hline Diciembre & Sur & 2 & A & 13 & Algal & 72 & - \\
\hline Diciembre & Sur & 3 & A & 13 & Algal & 71 & - \\
\hline Diciembre & Sur & 11 & A & 13 & Algal & 71 & - \\
\hline Enero & Norte & 1 & A V & 18.7 & Algal & 90.8 & 27.25 \\
\hline Enero & Norte & 1 & A & 18.7 & Algal & 90.8 & 27.25 \\
\hline Enero & Norte & 3 & A & 18.7 & Mud & 90.8 & 27.25 \\
\hline Enero & Norte & 10 & A & 19.1 & Mud & 89.5 & 27.65 \\
\hline Enero & Sur & 2 & A D & 20 & Mud & 86.2 & 8.6 \\
\hline Enero & Norte & 2 & A D & 18.9 & Algal & 50.7 & 19.35 \\
\hline Febrero & Norte & 1 & A D & 16.7 & Sand & 90.5 & 21.2 \\
\hline Febrero & Norte & 1 & V A & 18.7 & Sand & 86 & 14.9 \\
\hline Febrero & Norte & 17 & A & 18.7 & Algal & 86 & 14.9 \\
\hline Febrero & Norte & 6 & A & 19.4 & Algal & 79.2 & 10.75 \\
\hline Febrero & Norte & 2 & A V & 17.6 & Algal & 87.7 & 3.6 \\
\hline Febrero & Norte & 20 & A & 24.3 & Algal & 60.1 & 7.95 \\
\hline Marzo & Norte & 9 & A & 20.8 & Algal & 83.4 & 20.1 \\
\hline Marzo & Norte & 3 & A & 23.9 & Algal & 77.6 & 11.7 \\
\hline
\end{tabular}


sitios donde no se observó a la especie. Los invertebrados encontrados en las muestras fueron principalmente de larvas de las familias Chironomidae y Ephydridae (ambas del Orden Diptera) en diferentes estadios, además de que se observó material orgánico no determinable.

La diversidad simpátrica de las parvadas asociadas a $C$. melodus durante el invierno estuvieron comprendidas por dos familias, Charadriidae, dos especies: C. alexandrinus y C. semipalmatus; y Scolopacidae, nueve especies: Tringa melanoleuca, Arenaria interpres, Calidris alba, C. pusilla, C. mauri, C. minuta, C. minutilla, C. alpina y Limnodromus griseus.

\section{DISCUSIÓN}

El área de estudio de Boca Ciega, Tamaulipas, México, es una de las áreas invernales de C. melodus, que está catalogada en Peligro de extinción (SEMARNAT 2010). Los sitios utilizados por C. melodus, corresponden a las zonas inundables por las mareas de las aguas del Golfo de México y de la Laguna Madre, de acuerdo con las épocas del año. Sin embargo, en invierno los fenómenos conocidos como "Nortes" son los que mantienen esta área en condiciones para la especie referida. De acuerdo con German García (com. pers.) observó la presencia de la especie anualmente en Boca Ciega desde el 2006 en ambos lados del canal.

Estudios previos mencionan la presencia invernal de C. melodus en la Laguna Madre (Mabee et al. 2001, Haig \& Oring 1985, Nicholls \& Baldassarre 1990a, Zonick 2000, Drake et al. 2001), y con este trabajo se corrobora, a diferencia de la Manifestación de Impacto Ambiental, donde no se reporta la presencia de la especie para Boca Ciega, solamente se menciona como posible (Anónimo 2004).

Se observaron individuos de C. melodus, así como de otras especies, alimentándose en las zonas inundables de esta localidad. $\mathrm{La}$ técnica de alimentación mayormente empleada por esta especie fue la visual (Pienkowski 1979), aunque en ocasiones se observó usando sus patas para la búsqueda del alimento, rasgando el suelo para después picotear. La especie siempre se encontró alimentándose en lugares húmedos en las orillas de los charcos de agua, sin mojarse, desplazándose a sitios cercanos sin volar (Cairns 1977).

Las pruebas de Cochran y Kendall presentaron una alta significancia de la especie con un tipo de sustrato y las pruebas de signos utilizando una distribución binomial indicaron que existe una alta preferencia hacia un tipo de sustrato. Esto sucede porque la especie al momento de estar alimentándose utiliza con mayor frecuencia el tipo de sustrato mud y algal, lo cual concuerda con lo mencionado por Haig (1992), Wilkinson \& Spinks (1994) y Haig \& Elliott-Smith (2004), donde la especie tiene preferencias por los sitios algal, mud y arena en playas, barras de arena e islotes, playas oceánicas y superficies de alga o arena protegidas por bahías, aunque no especifican la actividad realizada por el ave en cada sustrato.

Las muestras de sedimento indicaron la presencia de dos familias de dípteros: Chironomidae y Ephydridae. Estudios sobre la posible dieta de C. melodus incluyen moluscos, poliquetos, anfípodos, insectos y nematodos (Bent 1929, Nicholls 1989, Shaffer \& Laporte 1994, Nordstrom \& Ryan 1996, Zonick 2000). Shaffer \& Laporte (1994) mencionan que las aves consumen los mismos tipos de invertebrados en la misma proporción con que estén disponibles en la localidad. Además, Nordstrom \& Ryan (1996) en los lagos alcalinos de los Grandes Lagos, obtuvieron que del 45-99\% de la abundancia de invertebrados está compuesta por las familias Chironomidae y Ephydridae; Nordstrom \& Ryan (1996) en Dakota del Norte, mencionan un $95 \%$ de estas familias en su dieta. En las dos muestras tomadas en sitios donde no se observó a la especie alimentándose, se determinaron las mismas especies de dípteros, lo que comprueba la homogeneidad de presas en el área de estudio. Durante el invierno las aves playeras deben recargar las reservas alimenticias que perdieron durante la migración en el otoño para sobrevivir el invierno, y regresar a reproducirse en la primavera (Blem 1990). Por lo que la Laguna Madre es un sitio importante, al proporcionar áreas de 
alimentación y descanso para especies migratorias (DUMAC 1993).

Las parvadas en el área de estudio, de las cuales formaba parte del C. melodus fueron mayores a 100 individuos; sin embargo, la asociación presente de acuerdo a su hábitat son las aves playeras de zonas húmedas lodosas y planas, ya que prefieren áreas que no tengan agua en su superficie, pero que hayan sido humedecidas recientemente por el agua de las mareas (Haig \& Elliott-Smith 2004).

En este estudio se observó que la zona norte presentó mayor abundancia (80 individuos) que en la zona sur (19 individuos), siendo en esta última donde el flujo del agua fue bloqueado por la creación de una barrera de arena del dragado del canal. La oficina del U.S. Fish and Wilflife Service $(2008,2009)$ expone que el dragado es una de las causas de la destrucción, modificación o reducción de los hábitats para C. melodus. Aunque la Manifestación de Impacto Ambiental declara que la construcción de isletas y el dragado podrían favorecer el establecimiento de ciertas especies (Anónimo 2004), la mayoría de estas son especies que no requieren un hábitat específico y no se encuentran bajo ningún estatus de protección de acuerdo a la NOM.059-SEMARNAT-2010 (SEMARNAT 2010), como es el caso de la especie de este estudio. Debido a las actividades que se puedan desarrollar durante el año en el sitio, los autores recomendamos el desarrollo de estudios de aves de al menos un año de duración, y no solo una estación, ya que no es posible identificar el inventario total de aves del área o de aquellas especies migratorias o veraniegas que lo utilizan como área de paso o reproducción, y que están bajo algún estatus de protección, como la especie estudiada. Sería de importancia continuar evaluando el impacto que las actividades antropogénicas causan sobre C. melodus y otras especies.

\section{AGRADECIMIENTOS}

A Pronatura Noreste A.C. y Laboratorio de Ornitología de la Facultad de Ciencias Biológicas, Universidad Autónoma de Nuevo
León. Proyecto "Sitios hermanos para la conservación del Piping Plover y otras aves playeras en áreas de reproducción e hibernación en Norteamérica".

\section{RESUMEN}

Charadrius melodus es una especie migratoria en peligro de extinción. Cada año, la Laguna Madre recibe aves migratorias invernales, incluyendo a la especie antes mencionada, sin embargo su estabilidad se encuentra amenazada por el cambio de hábitat ocasionado por el dragado que se está realizando en la zona. La falta de estudios sobre C. melodus durante el invierno en la Laguna Madre, motivó la realización del trabajo (diciembre 2009-marzo 2010). Los objetivos fueron: 1) determinar la importancia del área invernal, 2) describir la etología y preferencias de sustrato en la alimentación, 3) analizar ítems alimenticios potenciales y describir la diversidad simpátrica asociadas a C. melodus. Se observaron 99 individuos durante el muestreo. Las pruebas de Cochran y Kendall presentaron una alta significancia de la especie con un tipo de sustrato y las pruebas de signos utilizando una distribución binomial indicaron que existe una alta preferencia hacia un tipo de sustrato. La mayor actividad registrada durante el periodo de muestreo fue la de alimentación. Los recursos alimenticios encontrados en las muestras de sedimentos fueron larvas de Dípteros. Las especies simpátricas a C. melodus pertenecen al orden Charadriiformes con dos familias: Charadriidae nueve especies y Scolopacidae dos especies. Por la importancia de la zona, se recomienda proteger Boca Ciega, pues representa sitios de alimentación, protección y descanso para el Chorlo melódico.

Palabras clave: Chorlo melódico, Boca Ciega, periodo invernal, Tamaulipas, Laguna Madre, México.

\section{REFERENCIAS}

Adams, C.G. 1984. Piping Plover at Lake Athabasca, Saskatchewan: a significant northward expansion. Canadian Field. Nat. 98: 59-60.

American Ornithologist's Union. 1998. Check-list of North American Birds. American Ornithologit's Union, Washington, D.C., USA.

Anónimo. 2004. Estudio de Factibilidad, Proyecto Ejecutivo y Manifestación de Impacto Ambiental para la Construcción de Escolleras y Dragado en Boca Ciega, Tamaulipas. México.

Bent, A.C. 1929. Charadrius melodus. U.S. Nat. Mus. Bull. 146: 236-246.

Blem, C.R. 1990. Avian energy storage, p. 59-113. In R.F. Johnston (ed.). Current Ornithology Vol. VII, Plenum, New York, USA. 
BirdLife International. 2008. Charadrius melodus. In IUCN 2010. IUCN Red List of Threatened Species. Version 2011.2. (Consultado: 2 Marzo 2012, www. iucnredlist.org).

Boyd, R. 1991. First nesting record of the Piping Plover in Oklahoma. Wilson Bull. 103: 305-308.

Brown, S. 1987. Comparative breeding biology of the piping plover and spoted and pipier. Master Thesis, University of Michigan, Ann Arbor, Michigan, USA.

Cairns, W.E. 1977. Breeding biology and behavior of the Piping Plover (Charadrius melodus) in southern Nova Scotia. Master Thesis, Dalhousie University Halifax, Nova Scotia, Canadá

Cairns, W.E. \& I.A. McLaren. 1980. Status of the Piping Plover on the east Coast of North America. American Birds 34: 206-208.

Drake, K.C., J.E. Thompson \& K.L. Drake. 2001. Movements, habitat use, and survival of nonbreeding Piping Plovers. Condor 103: 259-267.

DUMAC. 1993. Informe final ecogeográfico para el manejo y conservación de la laguna madre, Tamaulipas y zona de influencia. Ducks Unlimited de México, A.C., Monterrey, Nuevo León, México.

Farmer, M. \& E. Carrera-González. 1993. Mid-sinter surveys of Reddish Egret foraging habitat in the Laguna Madre, Mexico. Report to US/Mexico Joint Committee of U. S. Fish and Wildlife Service and Secretaría de Desarrollo Social. Albuquerque, Nuevo México, USA.

Flemming, S.P., R.D. Chiasson, P.C. Smith, P.J. AustenSmith \& R.P. Bancroft. 1988. Piping Plover status in Nova Scotia related to its reproductive and behavioral responses to human disturbance. J. Field Ornithol. 59: 321-330.

Haig, S.M. 1992. Piping Plover (Charadrius melodus), p. 18. In A. Poole, F. Stettenheim \& E. Gill (eds.). The birds of North America, no. 2, Academy of Natural Sciences, Philadelphia, and American Ornithologists Union, Washington, D.C., USA.

Haig, S.M. \& E. Elliott-Smith. 2004. Piping Plover (Charadrius melodus), The Birds of North America Online (A. Poole, Ed.). Ithaca: Cornell Lab of Ornithology; Retrieved from the Birds of North America Online.

Haig, S.M. \& L.W. Oring. 1985. Distribution and status of Piping Plover throughout the annual cycle. J. Field Ornithol. 56: 334-345.

Haig, S.M. \& L.W. Oring. 1987. Population studies of Piping Plovers at Lake of the Woods, Minnesota, 1982-87. The Loon 59: 334-345.

Haig, S.M. \& L.W. Oring. 1988a. Mate, site and territory fidelity in Piping Plovers. Auk 105: 268-277.

Haig, S.M. \& L.W. Oring. 1988b. Distribution and dispersal in the Piping Plover. Auk 105: 630-638.
INEGI. 2000. Cuaderno Estadístico Municipal. Instituto Nacional de Estadística y Geografía, San Fernando, Tamaulipas, México.

Jonhnson, C.M. \& G.A. Baldassarre. 1988. Aspects of the wintering ecology of Piping Plover in coastal Alabama. Wilson Bull. 100: 214-233.

Labougle, J.M., A. Contreras-Arquieta, A. Banda-Valdez, M.A. Cruz-Nieto, W.E. Salinas-Castillo \& J.A. Campos-Flores. 2002. Laguna Madre Tamaulipas: Estudio Previo justificado para proponer la región, como área natural protegida. Informe Final. CONANP, México.

Lanza-Espino, G. 1991. Oceanografía de Mares Mexicanos. AGT Editor, México, D.F., México.

Martínez, M.R. 1978. Problemática actual de la Laguna Madre de Tamaulipas que impide el desarrollo de prácticas acuaculturales y soluciones posibles. Mem. $2^{\circ}$ Simp. Latinoam. Acuacult. 3: 2497-2507.

Mabee, T.J., J.H. Plissner, S.M. Haig \& J.P. Goossen. 2001. Winter distributions of North American plover in the Laguna Madre regions of Tamaulipas, Mexico and Texas, USA. Wader Study Group Bull. 94: 39-43.

MacIvor, L.H. 1990. Population dinamics, breeding ecology, and management of piping plover on outer Cape Cod, Massachusetts. University of Massachusetts, Amherst, Massachusetts, USA.

Melvin, S.M., C.R. Griffin \& L.H. MacIvor. 1991. Recovery strategies for piping plovers in managed coastal landscapes. Coast. Manage. 19: 21-34.

Merritt, R.W. \& K.W. Cummins. 1996. An introduction to the aquatic Insects of North America. Kendall/Hunt, Dubuque, Iowa, USA.

Nicholls, J.L. 1989. Distribution and other ecological aspects of Piping Plover (Charadrius melodus) wintering along the Atlantic and Gulf Coasts. Auburns University, Alabama, USA.

Nicholls, J.L. \& G.A. Baldassarre. 1990a. Winter distribution of piping plovers along the Gulf and Atlantic Coast of the U.S. Wilson Bull. 102: 400-412.

Nicholls, J.L. \& G.A. Baldassarre. 1990b. Habitat selection and interspecific associations of Piping Plovers along the Atlantic and Gulf Coasts of the United States. Wilson Bull. 102: 581-590.

Nordstrom, L.H. 1990. Assessment of habitat suitability for restablishment of Piping Plover in the Great Lakes National Lakeshrores. University of Missouri, Columbia, USA.

Nordstrom, L.H. \& M.R. Ryan. 1996. Invertebrate abundance at occupied and potential Piping Plover nesting beaches: Great Plains alkali wetlands vs. the Great Lakes. Wetlands 16: 429-435. 
Patterson, M.E., J.D. Fraser \& J.W. Roggenbuck. 1991. Factors affecting Piping Plover reproductivity on Assateague Island. J. Wildlife Manage. 55: 525-531.

Pienkowski, M.W. 1979. Differences in habitat requirements and distributional patterns of plovers and sandpipers as investigated by studies of feeding behavior. Verh. Orn. Ges. Bayern 23: 105-124.

Prindiville-Gaines, E. \& M.R. Ryan. 1988. Piping plover habitat use and reproductive success in North Dakota. J. Wildlife Manage. 52: 266-273.

Powell, A.N. \& F.J. Cuthbert. 1992. Habitat and reproductive success of Piping Plovers nesting on Great Lakes islands. Wilson Bull. 104: 155-161.

Ridway, R. 1919. The birds of north and middle America. Part VIII. Smithsonian Institution. United States National Museum. Government Printing Office, Washington, USA.

Root, B.G., M.R. Ryan \& P.M. Mayer. 1991. Piping Plovers survival in the Great Plains. J. Field Ornithol. 63: $10-15$.

SEMARNAT. 2010. Norma Oficial Mexicana NOM059SEMARNAT-2010. Protección ambiental- Especies nativas de México de flora y fauna silvestres-Categorías de riesgo y especificaciones para su inclusión, exclusión o cambio-Lista de especies en riesgo. 30.XII.2010.

SPP. 1983. Síntesis Geográfica del Estado de Tamaulipas. Secretaría de Programación y Presupuesto (SPP), Instituto Nacional de Estadística Geografía e Informática. México, D. F., México.

Strauss, E. 1990. Reproductive success, life history patterns, and behavioral variation in a population of
Piping Plovers subjected to human disturbance. Tufts University, Boston, Massachusetts, USA.

Schwalbach, M.J. 1988. Conservation of Least Terns and Piping plover along the Missouri River and its major tributaries in South Dakota. Thesis, South Dakota State University, Brookings, South Dakota, USA.

Shaffer, F. \& P. Laporte.1994. Diet of Piping Plovers on the Magdalen Islans, Quebec. Wilson Bull. 106: 531-536.

Sidle, J.G., D.E. Carlson, E.M. Kiersh \& J.J. Dinan. 1992. Flooding: mortality and habitat renewal for Least Terns and Piping Plovers. Colonial Waterbirds 15: 132-136.

U.S. Fish and Wildlife Service. 2008. Endangered species program. New Jersey Field Office, New Jersey, USA. (Consultado: 27 septiembre 2010, http://www.fws. gov/ northeast/njfieldoffice/Endangered/plover.html).

U.S. Fish and Wildlife Service. 2009. Piping plover (Charadrius melodus) 5 year review: summary and evaluation. Hadley, Massachusetts, USA.

Wilkinson, P.M. \& M. Spinks. 1994. Winter distribution and habitat utilization of piping plovers in South Carolina. Chat 58: 33-37.

Zabalegui, M. [XE “Zabalegui, M.L.M.”] \& F. Contreras. 1990. Hidrología, nutrientes y productividad primaria en la laguna Madre, Tamaulipas, México. Res. VIII Cong. Nal. Oceanog. Mazatlán, Sinaloa, México.

Ziewitz, J.W., J.G. Sidle \& J.J. Dinan. 1992. Habitat conservation for nesting least and Piping Plovers on the Platte River, Nebraska. Prairie National 24: 1-20.

Zonick, C.A. 2000. Winter ecology of Piping Plovers (Charadrius melodus) along the Texas Gulf Coast. $\mathrm{PhD}$ Thesis, Missouri University, Columbia, USA. 